\title{
Entre la grandeza cívica y la pequeñez económica: los regímenes de justificación en los fallos sobre reajuste de movilidad previsional en Argentina
}

\section{Between Civic Worth and Economic Meanness: Justification regimes in the ver- dicts on adjustments in pensions in Argentina}

\author{
Santiago Gabriel Calise ${ }^{1}$ \\ Universidad de Buenos Aires, Argentina
}

\begin{abstract}
RESUMEN Este artículo analiza tres fallos centrales concernientes a la falta de reajuste en la movilidad de los haberes previsionales en Argentina, desde la teoría de la justificación de Boltanski y Thévenot. En ellos se contraponen argumentos desde el mundo cívico e industrial. Desde el mundo industrial se cuestiona la forma de atribución de grandeza utilizada en la sociedad y se busca mostrar la pequeñez y mezquindad del jubilado. Jurídicamente hablando, se apela a la semántica de la crisis o emergencia económica para justificar la suspensión del derecho constitucional a la movilidad jubilatoria. Por el contrario, desde el mundo cívico se pone énfasis en el esfuerzo patriótico para el desarrollo del país realizado por el jubilado en sus años de actividad. Se considera que el derecho a la movilidad tiene un contenido humanitario y social, y no solamente económico. Los años de aportes son años de acumulación de grandeza, de sacrificio. La proporcionalidad "razonable", "necesaria” y/o "justa" es el concepto clave que indica qué relación debe existir entre los años de trabajo, la grandeza conseguida, y el haber jubilatorio percibido.
\end{abstract}

PALABRAS CLAVE Movilidad jubilatoria; emergencia económica; proporcionalidad; disputa; diferendo.

1. Investigador del CONICET-Universidad de Buenos Aires. Instituto de Investigaciones Gino Germani (IIGG). Buenos Aires, Argentina. Email: c_santiago_g2000@yahoo.com.ar 
ABSTRACT This paper analyzes three central verdicts in the area of pension adjustments in Argentina, utilizing Boltanski and Thévenot's justification theory. In these cases, arguments from the civic world are opposed to arguments from industry. The industrial world questions how society attributes worth, attempting to demonstrate the meanness and pettiness of pensioners. Legally speaking, the semantics of crisis or economic emergency are invoked to justify suspension of the constitutional right to an adjustable pension. In the civic world, by contrast, the emphasis is placed on pensioners' patriotic efforts to the forward country's development during their active years. The right to an adjustable pension is considered to have a humanitarian and social, and not only an economic content. The years when the pensioner was paying contributions were years of worth accumulation and sacrifice. "Reasonable", "necessary" and/or "fair" proportionality is a key concept, indicating the relationship that should exist between years of work, worth achieved and retirement income.

KEYWORDS Pension adjustability; Economic Emergency; Proportionality; Dispute; Disagreement.

\section{Introducción}

Durante las últimas décadas, el sistema previsional en Argentina fue objeto de continuos procesos de reforma y contra-reforma. A mediados de los años noventa se produjo el pasaje del sistema previsional público al privado ${ }^{2}$. Estudios posteriores ${ }^{3} \mathrm{se}$ encargaron de reevaluar el impacto de la reforma sobre el conjunto de la sociedad, y sobre los jubilados en particular. Por su parte, Goldberg y Lo Vuolo ${ }^{4}$ estudiaron la historia del sistema previsional argentino y relacionaron el modelo de acumulación económica de la década de los noventa con la reforma del sistema. Respecto del Sistema Integrado de Jubilaciones y Pensiones y el régimen de capitalización, García Rapp 5 analiza su implementación y evolución, evaluando las causas de los malos resultados obtenidos, especialmente durante la crisis de 2001-2002.

Luego de la crisis económico-social de 2001-2002 que sufrió Argentina, se abrió el paso para nuevas transformaciones. Los trabajos de Arza ${ }^{6}$ y de Danani y Beccaria ${ }^{7}$ ponen la atención en las reformas y contra-reformas que sufrió el sistema previsional

2. ISUANI et al. (1996); ISUANI y SAN MARTINO (1995b); ISUANI y SAN MARTINO (1995a).

3. FISCELLA (2005); HUJO (2004).

4. GOLDBERG y LO VUOLO (2006).

5. GARCÍA RAPP (2004).

6. ARZA (2009; 2012a; 2012b; 2012c).

7. DANANI y BECCARIA (2011). 
durante los últimos quince años, con el retorno del control estatal sobre las jubilaciones, analizando cuestiones como la ampliación de la cobertura y las moratorias

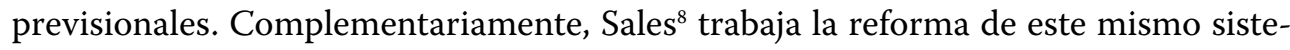
ma, ocurrida en el año 2007, mostrando las mejoras que este cambio ha conllevado. Beccaria y Danani ${ }^{9}$, nuevamente, analizando el proceso de judicialización de los conflictos previsionales, señalan algunos aspectos negativos, como el activismo judicial que no permite las acciones de clase, que transformarían los beneficios individuales en colectivos, y la redefinición de la cuestión previsional en términos de expertos judiciales, sustrayéndolo del debate público. De esta manera, se limitaría el alcance y legitimidad de los beneficios obtenidos en el período de posconvertibilidad.

Panigo y Médici $^{10}$ analizan, en una perspectiva histórica, los cambios ocurridos a partir de 2003 en el sistema previsional argentino, con la adopción de un sistema mixto, haciendo énfasis en las políticas inclusivas de esta transformación (entre ellas la AUH, la eliminación del régimen de capitalización y el Programa de Inclusión Previsional). En esta misma línea de trabajo, Vaca y Veritier ${ }^{11}$ estudian la implementación del Fondo de Garantía de Sustentabilidad y la Asignación Universal por Hijo, mostrando su impacto positivo en el crecimiento económico, las inversiones y el consumo. En otros trabajos ${ }^{12}$ se observa que, producto de las medidas tomadas entre 2004 y 2006, las políticas de seguridad social ampliaron la cobertura y redujeron la pobreza, pero también mejoraron la calidad de vida, la educación, la salud y la longevidad.

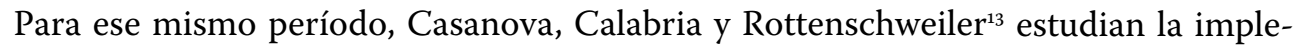
mentación de la moratoria previsional, que aumentó la cobertura del sistema al 90\%, también mostrando las fuentes de financiamiento del programa.

Messina ${ }^{14}$ examina este mismo período, destacando los aspectos positivos de las transformaciones ocurridas (ampliación de la cobertura, aumento del poder adquisitivo), al tiempo que recalca que la dualidad del mercado laboral argentino impide el correcto funcionamiento del sistema previsional. Por su parte, Agú ${ }^{15}$ destaca que las políticas de inclusión aplanaron la distribución del ingreso de los jubilados, tornándola más progresiva. Sin embargo, si no se realizan nuevas modificaciones, el sistema volverá a recuperar su fisonomía originaria de cobertura reducida. Por su parte,

8. SALES (2008).

9. BECCARIA Y DANANI (2012).

10. PANIGO y MÉDICI (2013).

11. VACA y VERITIER (2012).

12. BENIGNI et al. (2012); D'ELIA (2007); D'ELIA et al. (2011).

13. CASANOVA et al. (2014).

14. MESSINA (2014).

15. AGÚ (2015). 
Bellina Yrigoyen ${ }^{16}$ muestra cómo a partir de 2008 se reduce la desigualdad entre los adultos mayores, aunque esto no entraña un significado positivo, ya que se debió a la falta de actualización de los beneficios jubilatorios superiores a la mínima.

Como puede observarse, los trabajos antes citados se han centrado en los efectos positivos que han beneficiado a un amplio conjunto de adultos mayores, sobre todo, a través de los procesos de ampliación de la cobertura previsional. Por el contrario, solamente en una publicación se ha tratado el tema de la falta de actualización de las jubilaciones sufridas por cientos de miles de beneficiarios. Es por ello que este artículo se propone analizar tres fallos dictados por la Corte Suprema, que han marcado el camino de los reclamos por falta de reajuste en la movilidad de los haberes previsionales en Argentina. Siguiendo la definición proporcionada por la Corte Suprema de Justicia de la Provincia de Santa Fe, la "movilidad es la propiedad de que está dotada la jubilación, de variar su monto nominal de modo de conservar una razonable proximidad con el valor de la remuneración de que gozaría el agente en actividad"'17. La reforma constitucional de 1957, con la incorporación del artículo 14 bis, sancionó en Argentina el derecho a las “jubilaciones y pensiones móviles”. Desde aquella época, los conflictos judiciales entre el Estado y los jubilados y pensionados se sucedieron periódicamente. Ya en 1968, la Corte Suprema consideró confiscatoria la reducción de los haberes introducida por las leyes 18.037 y $18.038^{18}$, lo cual dio lugar al pago de actualizaciones ${ }^{19}$. Ante la imposibilidad de pagar, se sancionaron las leyes 23.928 y $23.982^{20}$, que prohibían la indexación o actualización de las deudas, y se emitieron los Bonos de Consolidación de las deudas previsionales (Bocon) a diez años de plazo para poder honrar las obligaciones. Una nueva reforma en $1994^{21}$ determinó que la movilidad estaría en función de las variaciones del Aporte Medio Previsional Obligatorio (AMPO). Sin embargo, un año más tarde, la ley $24.463^{22}$ derogó el mencionado artículo y determinó que la movilidad se establecería anualmente mediante la ley de presupuesto. De este modo, se desterró cualquier posibilidad de que los haberes de los pasivos guarden relación con los de los activos, que es la idea esencial que subyace al concepto de movilidad ${ }^{23}$. Peor aún es que el Congreso omitió en los sucesivos presupuestos toda referencia a la movilidad jubilatoria. Por lo tanto, por cuatro años, to-

\section{BELLINA YRIGOYEN (2015).}

17. FEO (2009) p. 19.

18. Ley 18.037 de 1968 y Ley 18.038 de 1969.

19. ALVAREZ CHÁVEZ (2008); PAYÁ y MARTÍN YÁÑEZ (2015).

20. Leyes 23.928 y 23.982 de 1991.

21. Ley 24.241, art. 7, de 1993.

22. Ley 24.463 de 1995.

23. ÁLVAREZ CHÁVEZ (2008). 
das las jubilaciones quedaron congeladas. A partir del año 2003, mediante sucesivos decretos $^{24}$ se incrementó el valor del haber mínimo solamente, y en $2006^{25}$ se otorgó un aumento del $10 \%$ para quienes cobraran menos de 1.000 pesos. Como consecuencia, la proporción de jubilados que recibían el haber mínimo pasó de $27 \%$ en 2001 a $63 \%$ en $2008^{26}$. Fue recién en 2008 , a través de la ley 26.417 , que se implementó una nueva metodología de cálculo de la movilidad, aunque no se subsanó el deterioro en las prestaciones generado por varios años de inflación.

Debido a que entre el período 2002-2006 gran parte de los jubilados no recibieron actualización alguna en sus haberes, al tiempo que la inflación comenzaba a aumentar, es que se empezaron a multiplicar las demandas judiciales solicitando la recomposición en las prestaciones y el pago de la movilidad no actualizada, extendiéndose hasta nuestros días. Tal es así que la ley 27.260 ${ }^{27}$, denominada "Programa Nacional de Reparación Histórica para Jubilados y Pensionados”, fue publicitada como un intento de solucionar este grave problema.

Respecto de la cantidad de juicios por movilidad correspondientes a los años 2002-2006, el único dato oficial que hemos podido conseguir deriva del Informe $\mathrm{N}^{\circ}$ 99 del Jefe de Gabinete a la Honorable Cámara de Diputados de la Nación (Jefatura de Gabinete de Ministros. Presidencia de la Nación 22 marzo 2017), donde se indica que al mes de julio de 2016 existían 443.406 juicios previsionales. Según el cálculo de Belina Yrigoyen ${ }^{28}$, en 2002 había 2.205.658 de jubilados y en 2007, 2.944.396, o sea, que entre el $15 \%$ y el $20 \%$ de los jubilados iniciaron un juicio. Este porcentaje de litigantes podría parecer reducido, en proporción al total de afectados, sin embargo, semejante cantidad de procesos ha servido para paralizar los tribunales de seguridad social y a la Corte Suprema de la Nación misma.

Por lo tanto, para poder comenzar a comprender desde un punto de vista sociológico la problemática de los juicios por reajuste de la movilidad del período 2002-2006, se propone analizar tres fallos centrales de la jurisprudencia en la materia, desde la teoría de la justificación elaborada por Boltanski y Thévenot ${ }^{29}$. El primero de ellos es el fallo Chocobar ${ }^{30}$, que no pertenece al grupo de reclamantes perjudicados por la situación acaecida entre 2002 y 2006, pero se hace necesario su análisis para com-

$24.391 / 03$ y $1194 / 03$, de $2003 ; 683 / 04$ y $1199 / 04$, de $2004 ; 748 / 05$ y $1273 / 05$, de $2005 ; 764 / 06$, de 2006.

25. Decreto 764/06, de 2006.

26. ÁLVAREZ CHÁVEZ (2008).

27. Ley 27.260 de 2016.

28. BELLINA YRIGOYEN (2010).

29. BOLTANSKI y THÉVENOT (1997).

30. Chocobar, Sixto Celestino c/ Caja Nacional de Previsión para el Personal del Estado y Servicios Públicos s/ reajustes por movilidad (1996). 
prender el proceso de justificación. Reduciendo su contenido a lo más esencial, en este fallo se encuentra que, frente al reclamo de un jubilado que solicitaba un reajuste según la ley 18.037, la mayoría de la Corte entendió que el sistema de movilidad de tal ley se encontraba derogado por la ley 23.928 (ley de convertibilidad). Esto se debería a que tal ley prohibía la indexación por precios, variaciones de costos o repotenciación con posterioridad al $1^{\circ}$ de abril de 1991 . Por su parte, en el fallo Sánchez ${ }^{31}$, también se reclamaba un reajuste, que fue negado en las dos primeras instancias, siguiendo el antecedente Chocobar. En su recurso de apelación ante la Corte Suprema, la actora argumentó que la ley de convertibilidad resultaba confiscatoria, al negársele la aplicación del régimen de movilidad establecido por la ley 18.037. En este caso, la mayoría hizo lugar al reclamo.

Por último, en el primer fallo Badaro de $2006^{32}$, el jubilado reclamaba un reajuste de su beneficio superior a los $\$ 1000$, al no haber percibido ningún aumento desde 2002. La Corte Suprema entendió que se veía afectado el derecho constitucional a la movilidad previsional garantizado por el artículo 14bis, ordenando que se reajustase la jubilación del reclamante. En 2007, ante el incumplimiento de lo establecido en el fallo precedente por parte de las agencias del Estado, la Corte volvió a expedirse ${ }^{33}$, declarando la inconstitucionalidad del art. 7, inc. 2 de la ley 24.463 y exhortando a los otros poderes del Estado a que solucionen el problema del congelamiento de los haberes de los jubilados y pensionados. Respecto del caso particular, estableció reajustar el beneficio del Sr. Badaro, entre el $1^{\circ}$ de enero de 2002 y el 31 de diciembre de 2006, según el índice de salarios, nivel general, elaborado por el Instituto Nacional de Estadísticas y Censos.

En lo que concierne a la estructura del artículo, a continuación se expondrán los puntos centrales de la teoría de la justificación elaborada por Boltanski y Thévenot, haciendo hincapié en los conceptos más relevantes para la investigación. Debido a que es un texto que no cuenta con una versión en castellano, se buscará detallar lo más concisamente posible la estructura de la teoría. A continuación se pasará al análisis de los fallos. En éste no se seguirán linealmente sus argumentos, ni tampoco se pondrá la atención en la lógica y los fundamentos jurídicos de los textos. Los conceptos centrales que guiarán el trabajo serán los de fórmula de inversión y de disputa. Por último, se presentarán las conclusiones.

31. Sánchez, María del Carmen c. Administración Nacional de la Seguridad Social (2005).

32. Badaro, Adolfo Valentín c/ ANSeS s/ reajustes varios (2006).

33. Badaro, Adolfo Valentín c/ ANSeS s/reajustes varios (2007). 


\section{La teoría de la justificación:}

\subsection{El modelo general}

Como señala Boltanski ${ }^{34}$, el libro De la justification ${ }^{35}$ marca la necesidad que tienen los actores de respaldarse en regímenes de justificación que instauran principios dotados de una validez intrínseca, cuando se ven implicados en situaciones donde sus juicios son sometidos a crítica. Estos regímenes sirven para apuntalar tanto los argumentos críticos, como las justificaciones, para sostener tanto el acuerdo como la crítica. Considerando las filosofías clásicas que buscan pensar la posibilidad de conformar un orden legítimo que repose sobre un principio de justicia, los autores han llamado a tales órdenes "ciudades" (cités). A su vez, también se parte del supuesto de que, en las modernas sociedades conviven diferentes regímenes de justificación en un mismo espacio. Una hipótesis fundamental referida a la constitución de ciudades es que ésta tiene un alcance general, pero que ella no es válida para todas las sociedades, sino solamente para aquellas marcadas por la filosofía política moderna. Los órdenes de la filosofía política, según Boltanski y Thévenot, son metafísicas, en la medida en que definen unas humanidades ligadas por un bien común que sobrepasa la felicidad particular de cada persona.

El modelo se desarrollará a través la relación entre seis axiomas (a) y cuatro hipótesis $(\mathrm{H})$. El primer axioma (a1) es el principio de humanidad común de los miembros de la ciudad, el cual supone que todos pertenecen de la misma manera a la humanidad. Este principio, en ausencia de toda diferenciación determina una metafísica política trivial de un único nivel lógico, que los autores denominan Edén ( $\left.\mathrm{H}_{1}\right)$, donde reinaría el acuerdo permanente de todos con todos. El segundo axioma, el principio de disimilitud (a2), excluye los Edenes y supone por lo menos dos estados posibles para los miembros de la ciudad. A su vez, esta diferenciación dará lugar a ciertas formas de justificación de las acciones y a pruebas para atribuir tales estados.

Con el fin de compatibilizar estas imposiciones, el modelo supone que todos los miembros tienen la misma potencialidad de acceder a todos los estados, que los autores designan como la dignidad común (a3). Por consiguiente, se define un modelo de humanidad de diferentes estados $\left(\mathrm{H}_{2}\right)$, que abre la posibilidad de acuerdos no-triviales y de desacuerdos, que quedan limitados a litigios respecto de la atribución de un estado a una persona, sin implicar inmediatamente un diferendo más profundo sobre la definición de esos estados. Tales disputas solamente se detendrán si se ordenan los estados (a4). Semejante orden entre estados, necesario para coordinar las acciones y justificar las distribuciones, se expresa mediante una escala de valor de los bienes

\section{BOLTANSKI (2012).}

35. BOLTANSKI y THÉVENOT (1997). 
y las suertes ligadas a esos estados, creándose así una tensión con el primer axioma. Por lo tanto, cuando la posibilidad de acceder a todos los estados no se ve asegurada, el orden constituido puede degenerar en un fraccionamiento de humanidades sin relación entre ellas.

De todas maneras, si se presupone la común humanidad (a1) y la igual posibilidad de acceso (a3) a los estados superiores, no se entiende por qué todos los miembros de la ciudad no gozan del estado supremo. Para explicar tal situación, se hace necesario apelar a una fórmula de inversión (investissement), la cual liga los beneficios de un estado superior a un costo o sacrificio exigido para su acceso, de manera que los beneficios se equilibran con cargas. Consecuentemente, la grandeza procura beneficios a las personas que acceden a ese estado, como también a los más pequeños que son comprendidos por los grandes y que encuentran en los grandes la posibilidad de crecer según su dignidad. Por consiguiente, la fórmula de sacrificio o de economía es la reguladora que suprime la tensión entre la común humanidad y la humanidad ordenada $\left(\mathrm{H}_{3}\right)$.

Este modelo de humanidad ordenada permite justificar una gama más amplia de acuerdos que el precedente, aunque la eventualidad de un acuerdo ampliamente admisible no se encuentra del todo asegurada, ya que las personas pertenecientes a los estados inferiores, deseosas de aprovechar de los beneficios ligados a los estados superiores, tenderán, más que a soportar el costo que implica el acceso al estado superior (a5), a cuestionarlo. Una hipótesis complementaria $\left(\mathrm{H}_{4}\right)$ sostiene el modelo, y supone que la felicidad, la cual se hace mayor al ascender hacia los estados superiores, genera provecho para toda la ciudad, lo cual constituye un bien común (a6). Esta última condición suplementaria permite hablar de un orden de grandeza. El bien común se opone al goce egoísta, que debe ser sacrificado para poder acceder a un estado de grandeza superior. El estado de grande se diferencia del estado de pequeño no solamente porque proporciona mayor bienestar a aquellos que pueden acceder a él, sino que también repercute sobre el bienestar de los pequeños, puesto que al goce egoísta de los pequeños se le agregan los beneficios de la grandeza de los grandes.

La importancia del estado de grande es fundamental en este modelo, ya que la ciudad se identifica mediante el estado de grande y acceder al estado de grande implica ser identificado con la ciudad. En un modelo de ciudad semejante $\left(\mathrm{H}_{4}\right)$, se entremezclan las nociones de grandeza (a4) y de bien común (a6), unificadas bajo el principio común superior. Este principio permite contener los desacuerdos dentro de lo admisible, evitando que degeneren, poniendo en duda el principio de acuerdo, o sea, la definición de los estados de grandeza y, por lo tanto, los fundamentos de la ciudad.

Consiguientemente, la estructura del modelo presupone dos exigencias fundamentales fuertemente antagónicas: la exigencia de una humanidad común, que supone una forma de identidad compartida por todos; $y$ una exigencia de orden de tal 
humanidad. La definición del bien común es la piedra angular de la construcción que debe asegurar la compatibilidad entre ambas exigencias.

\subsection{Las ciudades}

El modelo esbozado por Boltanski y Thévenot identifica seis tipos de ciudades: la ciudad inspirada, la ciudad doméstica, la ciudad de la opinión, la ciudad cívica, la ciudad mercantil y la ciudad industrial. La calificación de personas y cosas según un orden de grandeza conlleva el despliegue de las ciudades en mundos comunes. En su obra posterior sobre el nuevo espíritu del capitalismo, Boltanski, junto a Chiapello ${ }^{36}$, introducirán una nueva ciudad, la ciudad por proyectos. A continuación, solamente se caracterizarán las ciudades y mundos cívicos e industriales, que serán aquellos en torno de los cuales girará el trabajo, puesto que son los lugares centrales desde los cuales se construirán las justificaciones en los textos bajo análisis.

En la ciudad cívica la paz civil y el bien común reposan sobre la autoridad de un soberano majestuoso e imparcial situado por encima de los intereses particulares. Este soberano se encuentra desencarnado, su cuerpo político puede existir sin encarnarse en el cuerpo físico de un príncipe, de tal manera que él puede realizarse por la convergencia de las voluntades humanas, cuando los ciudadanos renuncian a su singularidad y se separan de sus intereses particulares, apuntando solamente hacia el bien común. En este sentido, las personas son grandes o pequeñas según si se las considera en cuanto particulares o en cuanto ciudadanos miembros del soberano, según si la voluntad que los hace actuar es singular u orientada hacia el interés general.

En el mundo cívico, no son las personas humanas las que acceden al estado de grandeza superior, sino las personas colectivas, que se componen por la reunión de las primeras. Estos seres colectivos también se encuentran comprendidos por otros colectivos de dimensión superior, donde el más inclusivo termina siendo la humanidad. Un ser también puede calificarse como grande, si se lo reconoce como representativo, lo cual le otorga autoridad dentro de una organización y le confiere la capacidad de ejercer un poder. Los representantes o delegados son más grandes en cuanto tienen la misión de traducir las aspiraciones de las masas. Tienen la capacidad de representar los intereses, o sea, de transformar los intereses particulares en un interés colectivo. Estos representantes pueden perder su estado de grandeza, si caen en lo particular a causa de una desviación. La legalidad configura un tipo de grandeza particularmente apreciada en este mundo, ya que la relación de grandeza se ejerce mediante formas legales que definen y limitan la representatividad según un dominio (político, sindical, etc.), un espacio y un tiempo.

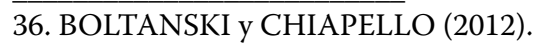


Los seres del mundo cívico poseen en sí mismos una aspiración que los lleva hacia lo común, hacia aquello que los une y que los incita a romper su aislamiento. La aspiración común a la unión define la dignidad de las personas. En este mundo, los seres son personas en cuanto susceptibles de tener derechos y obligaciones, o sea, en cuanto creados y autorizados por medio de un acto a través del cual se expresa la voluntad de todos. Ellos pueden acceder a la grandeza mediante el sacrificio de los intereses particulares e inmediatos, anteponiendo los intereses colectivos a los intereses individuales. La ciudad se derrumba cuando ella se abandona a lo particular. Cuando los seres no se ven fuertemente ligados mediante relaciones de solidaridad, se pierden y se dejan arrastrar por las desviaciones, disolviéndose en el individualismo.

Respecto de la ciudad industrial, los autores señalan que la ley fundamental del Estado es la regla contable del presupuesto. Siguiendo a Saint-Simon, Boltanski y Thévenot indican que el dinero es al cuerpo político lo que la sangre al cuerpo humano, por lo cual, la ley de las finanzas es la ley general de la cual todas las demás derivan o deben derivar. En este contexto, los grandes son aquellos que trabajan para descubrir y coordinar los hechos generales que servirían de base a todas la combinaciones de cultura, comercio o de la industria. Por el contrario, los pequeños son los menos inteligentes, aquellos cuyas ideas no van más allá de los asuntos domésticos. Los jueces de la grandeza industrial son los expertos, y la política es ciencia de la producción.

Por su parte, la organización del mundo industrial se basa en la eficacia de los seres, en su performance, en su productividad, en su capacidad para asegurar una función normal, en responder útilmente a las necesidades. La cualidad de grande la asumen los seres funcionales, operativos o profesionales, que expresan su capacidad para integrarse en los engranajes de una organización, al tiempo que su previsibilidad y su fiabilidad garantizan proyectos realistas para el futuro. Contrariamente, las personas pequeñas son las que no producen utilidad, que son improductivas, cuando proporcionan poco trabajo o proveen un trabajo de mala calidad, pero también lo son por no abrirse al futuro, por permanecer estáticas, rígidas, y no adaptarse. Además, las cosas son pequeñas en cuanto son subjetivas. Los grandes se relacionan con los pequeños por la responsabilidad que asumen en la producción, por el dominio que tienen sobre el futuro. La dignidad de las personas, la característica de la naturaleza humana en la que se basa este orden industrial es el potencial de actividad. Esa capacidad se expresa en un trabajo que representa el despliegue de la obra de la energía del hombre de acción.

Los objetos del mundo industrial son instrumentos, medios, movilizados por una acción que se presenta como una tarea de producción. Por otra parte, el progreso es la forma de inversión de este mundo, que se asocia a la operación de invertir que sopesa el precio del esfuerzo (en tiempo y dinero) y la rentabilidad a mediano plazo. Las inversiones le abrirán la puerta a un nuevo desarrollo. 


\subsection{El litigio, la disputa y el diferendo}

El estado de grandeza no se puede atribuir de manera permanente por la mera posesión de ciertas características personales, a causa del axioma de dignidad común (a3). La propiedad del modelo que otorga a todos los miembros de la ciudad la misma posibilidad de acceder a todos los estados genera incertidumbre sobre la medida de las grandezas, que se vuelve el punto de litigio cuando la disputa se inscribe en una ciudad. La concesión de un estado puede siempre ser puesta nuevamente en juego, al tiempo que la realización de la ciudad descansa sobre pruebas de grandeza que permiten la atribución de esos estados. Estas pruebas se apoyan sobre objetos exteriores, que sirven como instrumentos o aparatos de la grandeza, y que son particulares de cada ciudad, por lo cual, un instrumento que manifiesta la grandeza en una ciudad puede no ser tenido en cuenta en otra. Al mismo tiempo, cada ciudad tendrá su manera particular de construir las pruebas. El hecho de que la grandeza no esté ligada permanentemente a las personas permite la emergencia de litigios, o sea, desacuerdos respecto de la grandeza de las personas y, por lo tanto, sobre cuán justamente ésta se encuentra distribuida. En este sentido, un litigio llevará a constatar que la situación esté bien ordenada y a un reajuste de las grandezas, pero no pondrá en duda la naturaleza de los seres que tienen importancia.

A diferencia de los litigios, las disputas implican la refutación del origen de la prueba y no simplemente la invocación del efecto de circunstancias desfavorables para invocar la anulación o repetición. En apoyo de este tipo de reclamos, se esgrime la presencia, en la situación que les ha resultado perjudicial, de seres que no pertenecen al mundo en el cual debe realizarse la prueba para ser válida. Se busca valorizar seres de otra naturaleza, cuya injerencia introduce grandezas extranjeras a la prueba, que adolece de nulidad. Esta operación de desvelamiento extiende las posibilidades de desacuerdo, poniendo en duda el principio de la prueba e intentando sustituir la prueba en curso por una prueba perteneciente a otro mundo. De esta manera, se busca identificar los seres de otra naturaleza cuya presencia escondida degrada la prueba y desintegra el bien común denunciándolo como bien particular, para hacer valer el bien común de otra ciudad.

Bajo la forma del diferendo, la disputa no solamente avanza hacia la contestación de la relación entre el sacrificio consentido por la persona y el estado de grandeza al que ella accede, sino que también conlleva un cuestionamiento del bien común denunciado como simple autosatisfacción por oposición a otros principios de justificación (los grandes no realizan el bien común, sino solamente su propio bienestar; el trabajo que ellos llevan a cabo no es útil al bien común, solamente está al servicio de su vanidad o ambición personal, etc.). En el diferendo, el desacuerdo versará sobre la grandeza de los seres presentes, pero también sobre la identificación misma de los seres relevantes y de los que no importan, y, por lo tanto, sobre la verdadera naturale- 
za de la situación, sobre la realidad y sobre el bien común al que se puede hacer referencia para realizar el acuerdo. El objetivo no será el de rehacer la prueba de manera que ésta sea más pura y justa, por el contrario, se buscará desmitificar la prueba en sí misma, para poder ubicar las cosas en su verdadero terreno e instaurar otra prueba válida en un mundo diferente. Por lo tanto, la operación que busca mostrar que los supuestos grandes no son más que los pequeños del mundo actual, corresponde al primer momento del desvelamiento (la falsa grandeza esconde una miseria) que los autores nombran como crítica. La crítica implica que las partes no están de acuerdo sobre el mundo en el que debe llevarse a cabo la prueba para ser legítima. Para poder solucionar el diferendo, entonces, será necesario arribar a una prueba única para inclinar la situación hacia una naturaleza diferente, o para descartar los objetos sobre los que se apoyaba el desvelamiento, con el fin de fundar nuevamente la prueba en el mundo de origen.

\section{Los fallos sobre reajustes de la movilidad previsional: el choque entre la ciu- dad industrial y la ciudad cívica}

\subsection{La fórmula de inversión}

Uno de los movimientos argumentativos centrales que se realizan en el fallo Chocobar para comenzar a desplazar la prueba desde el mundo cívico, en el cual el derecho a la movilidad de las jubilaciones se encontraba inscripto, hacia el mundo industrial, es poner en duda la fórmula de inversión. En este fallo se considera que "un sistema de seguridad social no puede ser el resultado de políticas voluntaristas, sino el de las posibilidades ciertas, concretas y reales de una comunidad en un momento dado" ${ }^{37}$. Las posibilidades de una comunidad en un momento dado son los fondos provistos por el presupuesto, tal como se postula en la ciudad industrial. De esta manera se empieza anteponiendo una disponibilidad económica abstracta, que no se preocupa por discutir prioridades económicas, a la satisfacción de un derecho. A renglón seguido, se vuelve a apelar a la semántica de la crisis, considerando que la Argentina ha visto el fracaso de sucesivas leyes, que implicaban diferentes sistemas jubilatorios, durante todo el siglo XX. La razón de ello sería el aumento de los beneficiarios, pero sin la generación de los recursos para financiarlos, confiando en que el Estado afrontará los costos. Aquí se cuestiona la fórmula de inversión de los trabajadores, que, gracias a leyes supuestamente permisivas, han obtenido una prestación más alta de lo que le correspondería, si se tienen en cuenta los aportes realizados. Por otro lado, esta descompensación genera permanentes déficit, que parecerían no ser un problema para

37. Chocobar, op.cit., p. 77. 
la política. A continuación se interpela a gobernantes y gobernados para que tomen conciencia de la situación y se evite este tipo de errores en el futuro. No obstante, el magistrado prosigue con su crítica al sistema:

Que es un principio recibido que la jubilación debe ser sustitutivo equivalente del salario, que permita al beneficiario mantener en la pasividad un nivel de vida acorde con el que tenía en actividad. Tal objetivo se logra siempre y cuando el valor constante que el retirado recibe como prestación se hubiese formado sobre lo que realmente aportó. De lo contrario, aparecen inequívocas distorsiones, pues la relación se establece no ya entre el beneficio y lo aportado directamente por él durante toda su vida activa, sino entre la jubilación y lo que gane el trabajador que lo reemplace ${ }^{38}$.

$\mathrm{Al}$ poner en duda la fórmula de inversión, se cuestionan también los fundamentos del sistema jubilatorio de reparto. El juez insiste en la idea de que el jubilado debe recibir una prestación en estricta relación con lo aportado, y no con el nivel salarial que tenía en los años previos a su retiro. De esta manera, se impugna la justicia del sistema, debido a que la sociedad "debe desarrollarse en los límites de las previsiones presupuestarias pertinentes" ${ }^{39}$. Esto queda más claro, cuando el juez asevera que: "no se justifica que quien goza de buena salud y conserva su capacidad laboral, tenga derecho a exigir de los demás un esfuerzo superior al propio para que se le pague su prestación. Reconocer derechos para tales casos y mucho más sin contar con los medios adecuados para su resguardo, resulta una actitud pública y socialmente irresponsable"40. En este punto aparece claramente el concepto de esfuerzo, que implicaría que los jubilados, en general, cobran por sobre el esfuerzo realizado, o sea, gozan de una grandeza mayor a la que les correspondería sobre la base de la inversión efectuada en sus años activos, lo cual genera una sobrecarga sobre las generaciones activas, que se ven explotadas por los pasivos. Por consiguiente, se termina cuestionando la atribución de grandeza en la sociedad, considerando este desbalance entre esfuerzo realizado y monto de la jubilación actual como una actitud irresponsable.

En el fallo Sánchez, se buscará retornar la fórmula de inversión al mundo cívico, al cual pertenecía originariamente. Esto se observa explícitamente en el voto del Ministro Maqueda, quien hace propias las palabras de algunos convencionales de la reforma de la Constitución de 1957, para restituir al jubilado el status de grandeza que se había buscado degradar mediante el fallo Chocobar:

38. Ibid., p. 78.

39. Ibid., pp. 78-79.

40. Ibid., p. 79. 
En idéntico sentido, el convencional Riva manifestó que "hasta ahora siempre pareció una gracia lo que recibe el jubilado y pensionado. Pero no es así, no es una gracia del Estado, sino la retribución justa y amparadora por los servicios de toda una vida. El derecho a la jubilación es un verdadero derecho de propiedad que debe ser el premio a quien dio su esfuerzo por la patria, para que viva mejor. No puede retaceársele la retribución y así condenar a esos habitantes a vivir peor, como premio a los servicios prestados..." (Convención Nacional Constituyente 1957 - Diario de Sesiones, T. II, pág. 1371) ${ }^{41}$.

En este fragmento, el esfuerzo no se reduce al mero aporte dinerario que mensualmente realiza cada trabajador, sino que el esfuerzo tiene un contenido patriótico, ya que el trabajador no es un simple agente productivo y la sociedad no se restringe a la mera producción económica.

Finalmente, en el fallo Badaro, ante el deterioro de los haberes de los jubilados que cobraban más del beneficio mínimo, la fórmula de inversión vuelve a centrarse sobre el "esfuerzo contributivo". Dando la razón al reclamo masivo de los jubilados que vieron por años deteriorarse sus ingresos producto de la inflación, este fallo intenta recomponer la distribución de grandeza que les corresponde a los diferentes jubilados, según el propio esfuerzo contributivo. De este modo, se busca reestablecer la jerarquización de la grandeza propia del sistema jubilatorio de reparto, donde el mayor esfuerzo contributivo se ve ligado a una historia de ascenso laboral más exitosa, y que se ve recompensada con una mayor jubilación.

\subsection{Las disputas}

\subsubsection{La disputa por la definición de la movilidad}

\subsubsection{La movilidad como mero ajuste por inflación}

El intento de desplazar la prueba del mundo cívico al mundo industrial que se quiso llevar a cabo con el fallo Chocobar, implicó también una disputa sobre la definición del concepto de movilidad jubilatoria. En este sentido, el dictamen de la mayoría buscó reducir la movilidad a un simple ajuste por inflación que, en una situación de estabilidad de la moneda, como fue gran parte de los años 9o, implicaba el total congelamiento de los haberes jubilatorios. Para ello, la estrategia argumentativa tomó diferentes caminos. Uno de ellos es el debate hermenéutico de las discusiones de la Convención Constituyente de 1957. Respecto de este punto, la mayoría consideró que los convencionales asociaron directamente movilidad con ajuste por inflación,

41. Sánchez, op.cit., p. 11. 
sosteniendo que la movilidad era el remedio propuesto para el permanente proceso inflacionario que sufrió la economía argentina por décadas. Adicionalmente, estimó la mayoría que la falta de una definición más precisa del contenido de la garantía de movilidad presupone que se dejó explícitamente un amplio margen de maniobra al Congreso para reglamentarla, según su interpretación de lo que sea conveniente y posible para el sistema. De esta manera, el derecho a la movilidad se transforma en una decisión política sobre la distribución de los recursos escasos del Estado.

En segundo lugar, asociado al intento de ampliar la discrecionalidad del Congreso, aparece el argumento casi retórico que afirma que, con la eliminación del sistema de movilidad que proveía la ley 18.037 (que, según la argumentación de la mayoría, quedó derogada por la ley 23.928), y la no sustitución del mismo por otro sistema estructurado, implicaría una violación de la garantía constitucional expresada en el art. 14 bis. Pese a que esta interpretación estaría lesionando la garantía constitucional de la movilidad y la Corte misma sería consciente de ello, tal situación se justificaría porque la ley de convertibilidad se sancionó en el contexto de emergencia económica. Por lo tanto, se puede concluir que la situación de emergencia económica justifica que se suspendan ciertos derechos constitucionales. Lo que no se indica es por cuánto tiempo esta situación sería aceptable, puesto que la misma mayoría festeja la estabilización económica de ese momento, 1996, contra la sanción de la ley de convertibilidad de 1991. Es importante también resaltar que la cuestión de la suspensión de derechos producto de la emergencia económica implica también una discusión respecto de en qué consiste el bien general de la sociedad. La Corte asume un punto de vista apologético del accionar del gobierno menemista, presentando al gobierno como un actor preocupado por salvaguardar el bien común, haciendo posible la superación de la crisis económica y la eliminación de la inflación. Lamentablemente, en aras del bien común, algunos derechos patrimoniales pueden llegar a limitarse o suspenderse. Consecuentemente, se contrapone la grandeza del gobierno preocupado por el bienestar general, a la pequeñez de los reajustes reclamados por los jubilados, que, en un contexto de crisis, se resisten a contribuir, con un sacrificio, al bien común.

Un tercer movimiento argumentativo considera que los sistemas para medir la movilidad de los haberes previsionales (leyes 18.037 y 21.451) pierden su razón de ser producto de la sanción de la ley 23.928 (ley de convertibilidad) de 1991 y la consiguiente prohibición de las actualizaciones monetarias de las deudas. Debido a que los salarios dejaron de modificarse sistemáticamente producto de la variación del valor de la moneda, el régimen de movilidad queda sin sustento. $\mathrm{O}$ sea, puesto que se eliminó el problema de la inflación, la movilidad, en cuanto antídoto contra ella, también debe suprimirse, a riesgo de generar resultados absurdos. Al Sr. Chocobar la mayoría de la Corte Suprema le responde que, al realizar su reclamo, prescinde de considerar "esta 
nueva circunstancia"42 (la estabilización de la economía), y busca que le apliquen mecánicamente soluciones concebidas para otros contextos, que generarían aumentos que no reflejan la situación económica real. Esto significa que el jubilado estaría exigiendo un aumento que no le corresponde. De esta manera, se busca mostrar cierta mezquindad y pequeñez de los jubilados que reclaman el reajuste de sus haberes, que, en ciertas ocasiones, aprovechan de estos mecanismos para gozar de una grandeza que no les corresponde. Por consiguiente, se pone nuevamente en duda la fórmula de inversión de la grandeza cívica del jubilado, mostrándose su avidez económica, en ciertos momentos, injustificada.

\subsubsection{La movilidad como proporcionalidad}

En el voto en disidencia de los ministros Belluscio, Petracchi y Bossert se niega claramente que la garantía constitucional de la movilidad se reduzca solamente a un ajuste por inflación e indican que es una previsión "que tiene un profundo contenido humanitario y social que debe ser acatada por los tres poderes del Estado" ${ }^{43}$. De esta manera, al negar que la movilidad sea reductible a su mero aspecto económico, se busca negar que la prueba sea llevada al mundo industrial y abandone los parámetros del mundo cívico. En los sucesivos fallos Sánchez y Badaro, donde la prueba retornará al mundo cívico, quedará descartada la definición de la movilidad como ajuste por inflación y se consolidará la definición de la misma como proporcionalidad entre los ingresos de los pasivos respecto de los activos. Esto implica que, aunque no haya inflación, los ingresos de los jubilados deben seguir proporcionalmente los aumentos de los que gozan los ingresos de los activos. Esta proporcionalidad es calificada como "razonable”, "necesaria” y "justa”, aunque, en los textos no queda claro en qué consistiría tal razonabilidad o necesidad, cuáles serían sus límites, sino, solamente, que es potestad de los jueces discernir cuándo se ha cruzado semejante confín. La conclusión a la que llegan los Ministros disidentes en el fallo Chocobar es que, si en el período en discusión los salarios no estuvieron congelados (más allá de la falta de inflación), no hay razón para que las jubilaciones no acompañen ese aumento.

Adicionalmente, la Corte Suprema ha agregado, mediante el primer fallo Badaro de 2006, que la reglamentación de la movilidad debe reconocer "el derecho de los beneficiarios a una subsistencia decorosa y acorde con la posición que tuvieron durante su vida laboral" ${ }^{44}$. En este sentido, habría otra proporcionalidad más, que es la de mantener, mediante la jubilación, un nivel de vida similar al conquistado durante la vida laboral. Por lo tanto, esta definición de la movilidad como proporcionalidad con-

42. Chocobar, op.cit., p. 37.

43. Ibid., p. 99.

44. Badaro, Adolfo Valentín c/ ANSeS s/ reajustes varios (2006) p. 2. 
lleva implícitamente una fórmula de inversión que reconoce el sacrifico realizado durante los años trabajados, vistos como años de acumulación de grandeza. La sociedad no puede desconocer tal grandeza, forzando al jubilado a vivir en una injustificada pequeñez. Por lo tanto, el concepto de movilidad como proporcionalidad reproduce claramente un modelo jerárquico de acumulación de grandeza, que sostiene que la sociedad debe asegurar para sus mayores un nivel de grandeza similar al adquirido durante la vida laboral. Consiguientemente, el modelo es solidario intergeneracionalmente, pese a que es jerárquico y desigual al interior de la sociedad como un todo. Adicionalmente, atribuye al mundo laboral la condición de ser la principal fuente de grandeza.

En el fallo Chocobar, la mayoría aborda el concepto de proporcionalidad para poner en duda su verdadera pertinencia. Reduciendo el principio a mero "criterio interpretativo" y negándole el estatuto de "principios cardinales o axiomáticos, amalgamados a la cláusula constitucional de movilidad y, por ende, convirtiéndolos en una valla infranqueable para la razonable discreción del Congreso de la Nación”45, se busca circunscribir el concepto a un contexto histórico y textual muy acotado. Lo que se persigue es que el principio no bloquee el accionar del Congreso al momento de reglamentar los beneficios previsionales. Es más, los Ministros agregan que, a partir de la resolución de un conflicto interpretativo infraconstitucional, se genera un principio hermenéutico que sale de su cauce y se convierte en la única conclusión del texto constitucional. De esta manera, se compara al principio con un arma cargada, que con cada repetición incrusta el principio más profundamente en el derecho y el pensamiento, aplicándolo también a nuevos fines. Por otro lado, se califica al principio como "en cierto modo errático" ${ }^{46}$, ya que colisionaría con otros principios. El problema que se detecta aquí es el mismo que se expresaba más arriba, que limitaría al gobierno en su actividad legislativa, introduciendo postulados que no pueden ser alterados.

\subsubsection{Resolución de la disputa jurídica: el cambio de doctrina}

Las ideas centrales esgrimidas por los jueces disidentes en el fallo Chocobar se convertirán en los argumentos ratificados por la mayoría de los magistrados en el posterior fallo Sánchez, plasmando un cambio de doctrina que parece haber vuelto indiscutibles los argumentos de los magistrados disidentes. De esta manera, quedará establecido que, para el período 1991 a 1995 se deberá aplicar el criterio de movilidad establecido por la ley 18.037, eliminando el congelamiento de la movilidad determinado por el fallo Chocobar.

45. Chocobar, op.cit., p. 25.

46. Ibid., p. 26. 
Este cambio de doctrina implicó una victoria para los planteos realizados desde el mundo cívico. Más allá de los argumentos técnico-jurídicos, puede observarse también la referencia, mucho más marcada que en las justificaciones desde el mundo industrial, a principios jurídicos. Uno de éstos es el ya mencionado principio de razonabilidad o razonable proporcionalidad. Además, aparece otro principio de naturaleza más política, que es la doctrina in dubio pro justitia socialis, mencionada por los jueces disidentes en el fallo Chocobar y por el juez Maqueda en el fallo Sánchez. En este sentido, se cita directamente la formulación del fallo Berçaitz, de 1974, donde se puede leer:

"Y como esta Corte lo ha declarado, 'el objetivo preeminente' de la Constitución, según expresa su preámbulo, es lograr el 'bienestar general' (Fallos: 278: 313), lo cual significa decir la justicia en su más alta expresión, esto es, la justicia social, cuyo contenido actual consiste en ordenar la actividad intersubjetiva de los miembros de la comunidad y los recursos con que ésta cuenta con vistas a lograr que todos y cada uno de sus miembros participen de los bienes materiales y espirituales de la civilización. Por tanto, tiene categoría constitucional el siguiente principio de hermenéutica jurídica: in dubio pro justitia socialis. Las leyes, pues, deben ser interpretadas a favor de quienes al serles aplicadas con este sentido consiguen o tienden a alcanzar el 'bienestar', esto es, las condiciones de vida mediante las cuales es posible a la persona humana desarrollarse conforme a su excelsa dignidad" (Fallos 289:430, pág. 436) $)^{47}$.

El fragmento asocia directamente a la Constitución con el bienestar general y con la justicia social. La justicia social implica un ordenamiento de la actividad de los miembros y los recursos, conlleva una distribución de grandeza, de manera que todos puedan participar de ella. La justicia, en este sentido, no es una mecánica aplicación de la ley, ya que, como se encargan de explicar los Ministros en disidencia en el fallo Chocobar, aun si la ley de convertibilidad hubiese derogado la movilidad de los haberes establecida por el art. 53 de la ley 18.037, por mor del principio in dubio pro justitia socialis, se hubiese dejado sin efecto la derogación. En este sentido, la movilidad contribuye al bien común de la sociedad, y en pos de este bien común este principio de rango constitucional reestablecería la ley supuestamente abrogada. Este principio del mundo cívico, enfocado en la distribución, que subordina la situación económica por debajo de la justicia social, contrasta claramente con el mundo industrial, donde la ley de las finanzas hubiese sometido la movilidad a la disponibilidad económica y donde el acento estaría puesto en la producción y no en la distribución. En su voto en el fallo

47. Sánchez, op. cit., p. 14. 
Sánchez, el Ministro Maqueda vuelve a subrayar el concepto de dignidad, relacionando el derecho a la movilidad y su condición de derecho constitucional y el "principio de dignidad del haber previsional" ${ }^{48}$. Respecto del problema del bien común, en la decisión de la mayoría del fallo Chocobar puede observarse cómo la semántica de la crisis económica y la salvación de la patria se asocian a la reducción de los derechos jubilatorios. Si bien se reconoce que los beneficios jubilatorios concedidos no pueden caducar producto de una ley, sí puede verse afectado el monto de los haberes, producto de las "exigencias superiores de una política salvadora de su propia subsistencia, de su desenvolvimiento regular o por razones de interés colectivo que hacen al bienestar general, siempre que no resulten sustancial y arbitrariamente alterados (Fallos: 278:232 y sus citas)" 49 . Por lo tanto, según este argumento, se hace necesario el sacrificio de los jubilados, que deberán sufrir una limitación en su derecho, para poder salvaguardar el equilibrio económico. Así, contribuirían al bienestar general y al propio, ya que la reducción de los beneficios haría que los mismos se vuelvan pagables por parte del Estado, mientras que jubilaciones excesivamente onerosas contribuirían a agravar la crisis y, de esta manera, pondrían en peligro el pago de los haberes. En este párrafo, el énfasis está puesto en la posibilidad de limitar un derecho adquirido. Por el contrario, la argumentación desde el mundo cívico pone el acento en la intangibilidad del derecho adquirido a los haberes previsionales y a su movilidad. Este concepto de derecho adquirido es reforzado en el fallo Sánchez mediante los de derecho alimentario y el de la naturaleza sustitutiva del haber previsional. El primero indica que los beneficios previsionales tienen la función de otorgar una "cobertura de los riesgos de subsistencia y ancianidad, que se hacen manifiestos en los momentos de la vida en que la ayuda es más necesaria" ${ }^{\circ}$. Mediante mecanismos correctores, se busca aliviar la pequeñez y fragilidad del jubilado, reparar la injusticia y las contingencias de la vida. Por su parte, el segundo enfatiza la grandeza cívica del jubilado, puesto que la naturaleza sustitutiva se relaciona directamente con el "débito de la comunidad por el servicio prestado" ${ }^{1}$. Tanto por su grandeza cívica, como por su pequeñez económica, la argumentación desde el mundo cívico busca impedir la limitación al derecho de propiedad del haber previsional. Esta visión parece haber triunfado desde el punto de vista argumentativo en la doctrina jurídica, lo que no significa que se haya podido plasmar de manera generalizada en la recomposición de los haberes de todos los jubilados y pensionados afectados.

48. Ibid., p. 16.

49. Chocobar, op.cit., p. 39.

50. Sánchez, op.cit., p. 10.

51. Ibid. 


\subsubsection{La disputa política}

\subsubsection{El argumento económico}

Tanto el sistema previsional argentino como la economía del país han sufrido repetidas crisis económicas a lo largo del siglo XX y también en lo que va del siglo XXI. Ambas situaciones se encuentran íntimamente relacionadas en los reclamos de los jubilados y pensionados que se analizan en este trabajo. En estos fallos, como en muchas otras situaciones, las agencias del Estado han tomado el argumento de la emergencia / crisis económica como una justificación para suspender ciertos derechos o atribuirse ciertas capacidades de las que no podría gozar en una situación normal. Yendo más allá de los argumentos de naturaleza estrictamente jurídica, como los analizados en secciones anteriores, aparecen en los fallos problemas de carácter claramente político, relacionados con la capacidad de la Corte Suprema, especialmente, de ejercer el control de constitucionalidad. Este atributo que el ordenamiento jurídico le otorga, hace que ésta no sea solamente un tribunal, sino también un poder del Estado. Como indica el jurista Alberto Bianchi ${ }^{52}$, la Corte gobierna paralelamente a los otros poderes a través de sus pronunciamientos.

Siguiendo los fundamentos del mundo industrial, que ponen como ley fundamental a la regla contable del presupuesto, los Ministros que conformaron la mayoría en el fallo Chocobar elaboraron una estrategia argumentativa que aseguraba que, en función del bien común y producto de la situación crítica de la economía, ciertos derechos podían verse disminuidos. Un primer abordaje jurídico buscó apoyo en la Convención Americana de Derechos Humanos, arts. 22 y 26, para justificar el incumplimiento del derecho a la movilidad. En el primero de estos artículos se indica que los ciudadanos tienen derecho a la seguridad social "habida cuenta de la organización y los recursos de cada Estado". En el segundo artículo se indica que los Estados miembros se comprometen a actuar en pos de "la plena efectividad de los derechos que se derivan de las normas económicas, sociales y sobre educación...en la medida de los recursos disponibles". La conclusión que extrae la mayoría es que los recursos disponibles constituirían "una directriz adecuada a los fines de determinar el contenido económico de la movilidad jubilatoria, en el momento de juzgar sobre el reajuste de las prestaciones o de su satisfacción” ${ }^{33}$. Además, la mayoría del Supremo Tribunal señaló que la actualización de haberes debe permitir que el beneficiario mantenga el nivel de vida alcanzado durante la vida laboral, "sin perjuicio del análisis que, en orden a pautas concretas y demostradas, se efectúe respecto al estado financiero de los

52. BIANCHI (1992).

53. Chocobar, op.cit., p. 57. 
entes previsionales (Fallos: 305:2083 y 312:1706)" ${ }^{54}$. Por lo tanto, la movilidad quedaría subordinada a la disponibilidad económica de los entes del Estado, y la proporcionalidad se alcanzaría solamente si los recursos bastasen. Pero, debido a que el sistema previsional argentino es un régimen de reparto impuro, ya que utiliza fuentes de financiación de naturaleza impositiva y no solamente los aportes de los trabajadores, la cuestión de la "disponibilidad" es siempre una decisión política respecto de la asignación y utilización de los recursos del Estado. Esta interpretación restrictiva de la Convención Americana de Derechos Humanos será rechazada en el fallo Sánchez, donde se expresará que los tratados internacionales no pueden restringir o modificar derechos consagrados por la Constitución, siguiendo el art. 29 b de la misma Convención.

Más adelante, los Ministros harán referencia a la posibilidad de realizar, cuando las finanzas del sistema fallan sistemáticamente por años:

[U]na reforma general y reconstructiva impuesta por la necesidad de volver a poner las cosas en su quicio, equilibrando los egresos con los ingresos, que eche mano del recurso extremo de reducir los beneficios, actuales y futuros, dentro de una proporcionalidad justa y razonable, haciendo así efectivo el principio de solidaridad en que descansan estas instituciones, no puede ser objetada como arbitraria e inconstitucional. Lo justifica el interés público y la impone la conservación misma del patrimonio común de los afiliados ${ }^{55}$.

$\mathrm{El}$ argumento propuesto es que, en pos del bien común, todos los beneficiarios tendrían que hacer el esfuerzo de aceptar un ingreso menor, para poder equilibrar el sistema. Esta afectación de los derechos previsionales no sería ni arbitraria, ni inconstitucional. El problema aquí es que no se ha realizado una "reforma general y reconstructiva", puesto que la ley de convertibilidad no introdujo ningún sistema de movilidad, ni tampoco hizo referencia a ningún aspecto del sistema previsional. La ley 24.241, que implantó el "Sistema Integrado de Jubilaciones y Pensiones" sí implicó una reforma general, pero ella no estableció la reducción de los beneficios. Por consiguiente, cabe pensar que, a posteriori, y mediante una ley que nada expresaba sobre el derecho a la movilidad, se buscó recortar las prestaciones previsionales de manera velada. En vez de expresar claramente la necesidad de reducir las jubilaciones y pensiones por la crisis del sistema previsional, se habría optado por intentar congelar las prestaciones de manera indefinida, de modo que éstas se fuesen reduciendo paulatinamente.

54. Ibid., pp. 26-27.

55. Ibid., p. 38. 
Frente a este argumento que sostiene que un fallo en contra de las agencias del Estado llevaría a una crisis económica, el voto en disidencia en el fallo Chocobar indica que semejante razonamiento no es válido, ya que el legislador debió haber sopesado las consecuencias económicas de haber mantenido tales garantías constitucionales. Por lo tanto, aseguran los Ministros, "no es afectando derechos individuales de contenido alimentario en donde ha de encontrarse la solución al problema planteado" ${ }^{56}$.

El argumento de la crisis económica es casi un tópico de las agencias del Estado, a la hora de intentar no pagar sus obligaciones. Éste reaparecerá, precisamente, en el fallo Badaro de 2007. Superada la crisis de 2001-2002, el Estado seguirá argumentando la imposibilidad de otorgar la movilidad aludiendo a problemas económicos. En este caso, los magistrados refutarán este argumento indicando que no se ha demostrado la existencia de tales circunstancias económicas que impidieran el cumplimiento de la garantía constitucional de la movilidad. Por esto mismo, se desestimará el planteo de la ANSeS. Moderando su crítica, la Corte Suprema admite que, mediante decretos de necesidad y urgencia, se recompusieron los haberes de las prestaciones mínimas, priorizando la situación de los más pequeños. No obstante, tal respuesta no es suficiente para satisfacer la garantía conculcada.

\subsubsection{La discrecionalidad del Congreso}

Con el fin de otorgar mayor discrecionalidad al Congreso a la hora de disponer de los recursos económicos, el voto de la mayoría en el fallo Chocobar sostiene que el Poder Legislativo tiene la capacidad de reformular o modificar íntegramente el sistema de la seguridad social, incluida la movilidad de los haberes. Esta facultad debe ser de "gran latitud para la elección y empleo de esos medios" ${ }^{57}$, lo cual se relaciona con el hecho de que la Constitución admitiría que "cualquier opción racional es constitucional ${ }^{58}$ ". Semejante laxitud de los límites que impondría la Constitución al legislador sería justificada por la complejidad de su tarea de armonizar los intereses y necesidades de la Nación. En este sentido, se indica que la garantía de movilidad no es un concepto unívoco, que da lugar a una interpretación única e inmodificable, sino que es posible moldearlo y adaptarlo "a la evolución que resulte de las concepciones políticas, jurídicas, sociales y económicas dominantes que imperan en la comunidad en un momento dado" ${ }^{59}$.

56. Ibid., p.120.

57. Ibid., p. 18.

58. Ibid., p. 19.

59. Ibid., p. 17. 
Esta posición será duramente criticada en los fallos sucesivos. En el voto del Dr. Maqueda relativo al fallo Sánchez, el magistrado señala que la Constitución reconoce derechos efectivos y no ilusorios, por lo que el legislador no puede llenarlos de cualquier modo. La reglamentación de los mismos no puede alterarlos. De manera similar, en el fallo Badaro de 2007, se asevera que la ley 24.463 implicó la consagración de un régimen de movilidad con un nivel de protección menor que el anterior. En este sentido, se vuelve a reiterar que este derecho no es un enunciado vacío, sino que su fin es el de asegurar a los beneficiarios un nivel de vida acorde al que tuvieron durante su trayectoria laboral. En estos fallos se hará especial referencia al artículo 75, inciso 23, de la Constitución de la Nación Argentina, para enfatizar la protección especial de la que deben gozar los ancianos. Consecuentemente, se liga la movilidad con la garantía de igualdad de oportunidades y de trato, y con el goce de los derechos constitucionales. Además, en el fallo Badaro de 2007 se hará referencia al inciso 19 del artículo constitucional anteriormente mencionado, subrayando que el Congreso debe proveer lo conducente al desarrollo humano y al progreso económico con justicia social. De esta manera, vuelve a aparecer el principio de justicia social ligado al derecho a la movilidad, mostrando como el segundo contribuye a la realización del primero.

Esta discusión respecto de la modificabilidad y manipulabilidad de los derechos muestra cómo, para los argumentos desde el mundo industrial, éstos pueden adaptarse plásticamente a las interpretaciones y necesidades del gobierno, sin implicar un problema jurídico. Por el contrario, desde el mundo cívico, los derechos, especialmente constitucionales, son vistos de manera más rígida, enfatizando la necesidad de que éstos se cumplan efectivamente.

\subsection{Solucionar el diferendo}

Como se ha querido mostrar hasta aquí, dentro de la Corte Suprema se ha generado un diferendo jurídico-político respecto del problema del congelamiento de la movilidad de los haberes previsionales. El estudio de estos tres leading cases ha expuesto un cambio de doctrina que ha contrapuesto argumentaciones desde el mundo industrial y el mundo cívico. Estas últimas han terminado por primar, sin embargo, las agencias del Estado no han simplemente respetado lo expresado por la Justicia, especialmente en el caso Badaro. En el primer fallo Badaro de 2006 se insta a la ANSeS a que recomponga los haberes de los beneficiarios que cobran más que el mínimo. Pese a ello, la ANSeS hizo caso omiso, argumentando que la situación de emergencia económica hacía imposible otorgar semejante aumento. Como consecuencia, al año siguiente, la Corte Suprema vuelve a dictar otro fallo donde indica, ante la falta de respuesta del organismo estatal, cómo deberá realizarse la reestructuración. La Corte Suprema verificó que en el período que va del $1^{\circ}$ de enero de 2002 al 31 de diciembre de 2006, los beneficiarios con haberes superiores al mínimo recibieron un único aumento del 
$11 \%$ a través del decreto 764/o6. Por el contrario, los informes del INDEC (Instituto Nacional de Estadística y Censos) mostraron subas del 91,26\% en los precios y $88.57 \%$ en los salarios. Además, argumenta la Corte, que no se ha demostrado que semejante situación económica impida la reparación de la prestación del Sr. Badaro. Por consiguiente, se dispuso recomponer los haberes del actor siguiendo el nivel general del índice de salarios del INDEC. Como se encarga de destacar la Corte Suprema, estas conclusiones solamente afectan al caso concreto examinado. Frente al problema generalizado, y la consiguiente catarata de juicios del mismo contenido, la Corte exhorta a los otros poderes del Estado a reexaminar la situación y a dictar una ley para garantizar el derecho a la movilidad, ya que, en este caso, se decreta la inconstitucionalidad del art. 7 , inc. 2 , de la ley 24.463 :

24) Que en este entendimiento, esta Corte considera que contribuiría a dar mayor seguridad jurídica el dictado de una ley que estableciera pautas de aplicación permanentes que aseguren el objetivo constitucional. Una reglamentación prudente de la garantía en cuestión, además de facilitar el debate anual sobre la distribución de recursos y evitar el uso de facultades discrecionales, permitiría reducir la litigiosidad en esta materia, que ha redundado en menoscabo de los derechos de los justiciables y del adecuado funcionamiento del Poder Judicial (Fallos: 328:566 "Itzcovich"), por lo que se formula una nueva exhortación a las autoridades responsables a fin de que examinen esta problemática ${ }^{60}$.

Este considerando puede ser leído como un intento de buscar establecer un compromiso que realice el bien común, garantizando el derecho a la movilidad para todos los jubilados y pensionados, reduciendo la litigiosidad previsional, que ha afectado severamente al Poder Judicial. Mediante la ley 26.417 de octubre de 2008, el Congreso sancionó un nuevo sistema de movilidad de aumentos automáticos, que calcula la movilidad de acuerdo con una fórmula, por lo que no se dependerá de lo dispuesto en la Ley de Presupuesto de cada año. No obstante, el conflicto referido a la recomposición de los haberes producto del congelamiento de los años 2002-2006 no se ha resuelto. El Estado ha preferido seguir la estrategia de dejar que cada jubilado y/o pensionado realice su propio proceso judicial, en vez de otorgar una solución general. En este sentido, el Estado ha seguido argumentando y actuando desde la perspectiva del mundo industrial, evitando el pago siempre que fuese posible.

60. Badaro, Adolfo Valentín c/ ANSeS s/reajustes varios (2007) p. 9. 


\section{Conclusiones}

El estudio de estos tres leading cases ha mostrado un cambio de doctrina que ha desplegado argumentaciones de dos mundos, el industrial y el cívico. Uno de los conceptos centrales que proporciona la teoría de la justificación de Boltanski y Thévenot es el de fórmula de inversión. Éste relaciona los beneficios de un estado superior con costos o sacrificios necesarios para su acceso. En las argumentaciones desde el mundo industrial se busca mostrar al jubilado como un agente que goza de una grandeza superior a la que realmente le corresponde, cobrando prestaciones más altas de los aportes que realmente realizó al sistema jubilatorio. Por lo tanto, en general, se cuestiona directamente la forma de atribución de grandeza en la sociedad, y al sistema jubilatorio de reparto, sosteniendo que el jubilado debe cobrar estrictamente en función de los aportes realizados (como en los regímenes de capitalización). En particular, se busca mostrar la pequeñez y mezquindad del jubilado, que sigue reclamando más aumentos. La consecuencia de este desequilibro en la fórmula de inversión genera permanentes déficits para las finanzas del Estado. En este contexto, la ley fundamental de la sociedad debería ser la de desarrollarse en los límites indicados por las previsiones presupuestarias.

En lo que concierne al derecho a la movilidad previsional, las argumentaciones desde el mundo industrial buscan reducirlo a un simple ajuste por inflación, que libra a la interpretación del Congreso la manera de reglamentarlo. No obstante, el criterio principal a seguir a la hora de darle un contenido económico a este derecho es el de tener en cuenta los recursos disponibles. En realidad, estos dos principios (la movilidad como ajuste por inflación y la disponibilidad de recursos) solamente se vuelven compatibles en un contexto de baja o nula inflación, como durante el gobierno menemista. Consecuentemente, el primer criterio solamente indicaría el límite máximo de aumento posible, mientras el segundo sería el que terminaría por determinar los reajustes.

En el contexto de un modelo argumentativo, donde solamente la dimensión económica parece prevalecer, se apela a la semántica de la crisis o emergencia económica sufrida por la Argentina entre fines de los años '80 y principios de los '9o, para justificar la suspensión de ciertos derechos constitucionales, como la movilidad jubilatoria. Por consiguiente, en un contexto de crisis, durante el cual peligra el bienestar general, los reclamos por derechos patrimoniales individuales deben ser dejados de lado para atender al bien común. De esta manera, se vuelve a presentar al jubilado reclamante como mezquino y sumido en su mero interés particular.

Por el contrario, las argumentaciones desde el mundo cívico deslindan la fórmula de inversión del estricto cálculo matemático entre aportes y jubilaciones. El concepto principal es de esfuerzo patriótico realizado durante los años de trabajo para el desarrollo del país. Si en las argumentaciones desde el mundo industrial el régimen 
de capitalización, justamente introducido durante el gobierno de Menem, sería el modelo que reflejaría de manera más exacta la fórmula de inversión más justa, desde el mundo cívico se reivindica al modelo de reparto y su carácter solidario, donde cada jubilado cobraría en función del nivel de bienestar alcanzado durante la vida laboral. Desde este punto de vista, se considera que el derecho a la movilidad tiene un contenido humanitario y social, y no solamente económico. Los años de aportes son años de acumulación de grandeza, de sacrificio. Uno de los conceptos clave en esta interpretación es el de la proporcionalidad "razonable", "necesaria" y/o "justa" que debe haber entre los años de trabajo y la grandeza conseguida, y el haber jubilatorio percibido.

Por otra parte, a través del concepto de justicia social, se propone una visión del bien común muy distinta de la expresada desde el mundo industrial. La justicia social sería la más alta expresión de la justicia, que consiste en un ordenamiento de la actividad de los ciudadanos y de los recursos, con el fin de que cada uno participe de los bienes materiales y espirituales de la civilización. O sea, que se buscaría una inclusión total de todos, sin dejar a nadie excluido.

Por último, si en pos del bien común se les reclamaba a los jubilados un sacrificio desde el mundo industrial, desde el mundo cívico se indica que los jubilados no serían aquellos que deberían realizarlo. Esta idea toma las formas jurídicas de la intangibilidad del derecho adquirido a los haberes previsionales y su movilidad, del carácter alimentario de la jubilación y pensión, y de la naturaleza sustitutiva del haber previsional.

Desde el punto de vista argumentativo, este análisis de la jurisprudencia muestra que el cambio de doctrina ha conllevado el triunfo de los argumentos desde el mundo cívico en los fallos de la Corte Suprema de Justicia de la Nación. Si bien este cambio ha representado un triunfo del reclamo de tantos cientos de miles de jubilados, la disputa aún no se ha saldado completamente, ya que el Estado ha evitado solucionar el problema según las directivas indicadas por la Corte Suprema. Por consiguiente, el Estado, en las instancias judiciales, ha seguido argumentando y actuando desde la perspectiva del mundo industrial.

\section{Referencias bibliográficas}

AGÚ, Emanuel (2015): Algunas implicancias distributivas de las reformas del sistema previsional argentino, 2003-2013. Bariloche, Argentina. En: III Seminario Internacional sobre Desigualdad y Movilidad Social en América.

ALVAREZ CHÁVEZ, Víctor Hugo (2008): Sistema integrado previsional argentino y movilidad jubilatoria. Leyes 26,425 y 26,417 doctrina, jurisprudencia, modelos prácticos (Buenos Aires, García Alonso). 
ARZA, Camila (2009): Back to the State. Pension fund nationalization in Argentina. En United Nations Research Institute for Social Development, Ginebra, Suiza. Disponible en: http://citeseerx.ist.psu.edu/viewdoc/download?doi=10.1.1.621.3873\&r ep=rep1\&type $=$ pdf [fecha de consulta: 14 diciembre 2019].

ARZA, Camila (2012a): Extending coverage under the Argentinian pension system. Distribution of access and prospects for universal coverage. En International Social Security Review 65(2), pp.29-49. Disponible en: 10.1111/j.1468-246X.2011.01427.x [fecha de consulta: 14 diciembre 2019].

ARZA, Camila (2012b): The politics of counter-reform in the Argentine pension system. Actors, political discourse, and policy performance. En International Journal of Social Welfare 21(150), pp. 46-6o. Disponible en: 10.1111/j.1468-2397.2012.00872.x [fecha de consulta: 14 diciembre 2019].

ARZA, Camila (2012c): Who gets what? Distributional principles and impacts of the Argentine pension system. En Tempo do Mundo, 4(2), pp.183-205.

BECCARIA, Alejandra y DANANI, Claudia (2012): La política previsional argentina 2003-2011. balances, desafíos e incógnitas sobre la protección. En Argumentum 4(2), pp. 59-79. Disponible en: https://dialnet.unirioja.es/servlet/ articulo? codigo $=4835016$ [fecha de consulta: 13 noviembre 2019].

BELLINA YRIGOYEN, Jorge E. (2010): Evolución de la situación previsional de los adultos mayores en Argentina. (Período 1999-2007). En Invenio (24), pp. 73-99. Disponible en: http://www.ucel.edu.ar/upload/revista_invenio/Invenio_24_Junio_2010.pdf. [fecha de consulta: 29 julio 2018]

BELLINA YRIGOYEN, Jorge (2015): Los ingresos de los adultos mayores en Argentina según su nivel educativo en el período 1998-2013. En Invenio 18(35), pp.51-63.

BENIGNI, Mariana, LIEUTIER, Ariel y LUDMER, Gustavo (2012): Evolución reciente de sistema previsional argentino y su impacto en la pobreza de los adultos mayores. En Revista Debate Público. Reflexión de trabajo social, (4), pp. 197-214.

BIANCHI, Alberto (1992): Control de constitucionalidad. El proceso y la jurisdicción constitucionales (Buenos Aires, Ábaco).

BOLTANSKI, Luc y CHIAPELLO, Ève (2012): Le nouvel esprit du capitalisme (Paris: Gallimard).

BOLTANSKI, Luc y THEVENOT, Laurent (1997) : De la justification. Les économies de la grandeur (Paris, Gallimard).

BOLTANSKI, Luc (2012): Sociología y crítica social. Ciclo de conferencias en la Universidad Diego Portales (Santiago de Chile, Ediciones Universidad Diego Portales). 
CASANOVA, Luis, CALABRIA, Alejandro y ROTTENSCHWEILER, Sergio (2014): Financiamiento de la extensión de la previsión social. La experiencia Argentina desde una visión comparada y perspectivas futuras. $47^{\circ}$ Jornada Internacional de Finanzas Públicas, Córdoba, Argentina.

DANANI, Claudia y BECCARIA, Alejandra (2011): La (contra)reforma previsional argentina 2004-2008. aspectos institucionales y politico-culturales del proceso de transformación de la protección. En: Claudia DANANI y HINTZE, Susana (eds.) Protecciones y desprotecciones. La seguridad social en la Argentina, 199o-2010 (Los Polvorines Prov. de Buenos Aires Argentina, Universidad Nacional de General Sarmiento) pp. 103-152.

D'ELIA, Vanesa (2007): Pobreza en adultos mayores. evolución y determinantes a partir del Plan de Inclusión Previsional. XLII Reunión Anual de la AAEP, .Bahía Blanca, Argentina.

D'ElIA, Vanesa; ROTTENSCHWEILER, Sergio; CALABRIA, Alejandro; CALERO, Analía y GAIADA, Julio (2011): Análisis de la cobertura previsional del SIPA. protección, inclusión e igualdad. En Observatorio de la seguridad social, pp. 1-23. Disponible en: http://observatorio.anses.gob.ar/archivos/documentos/ An\%C3\%A1lisis\%2ode\%2ola\%2oCobertura\%2odel\%2oSIPA.pdf. [fecha de consulta: 14 noviembre 2019].

FEO, Armando F. J. de (2009): Movilidad jubilatoria. Actualidad del régimen nacional; régimen de la provincia de Santa Fe. (Santa Fe, Rubinzal-Culzoni).

FISCELLA, Sergio (2005): Estado, ciudadanía y política social. Estudio sobre los sistemas de jubilaciones y pensiones. (Buenos Aires, Espacio).

GARCÍA RAPP, Jorge (2004): El SIJP y el régimen de capitalización en el marco de la crisis argentina. En Revista Seguridad Social, 246, pp. 19-30.

GOLDBERG, Laura y LO VUOLO, Rubén M. (2006): Falsas promesas. Sistema de previsión social y régimen de acumulación. (Buenos Aires: Ciepp; Miño y Dávila Editores).

HUJO, Katja (2004): Reforma previsional y crisis económica: el caso argentino. En HUJO, Katja; MESA-LAGO, Carmelo y NITSCH, Manfred (eds.) Públicos o privados? Los sistemas de pensiones en América Latina después de dos décadas de reformas (Caracas: Nueva Sociedad), pp. 135-174.

ISUANI, Ernesto y SAN MARTINO, Jorge (1995a): El Nuevo Sistema Previsional Argentino. ¿Punto final a una larga crisis? (Segunda Parte). En Boletín Informativo Techint, 282, pp.43-67. 
ISUANI, Ernesto y SAN MARTINO, Jorge (1995b): El Nuevo Sistema Previsional Argentino. ¿Punto final a una larga crisis? En Boletín Informativo Techint, 281, pp.4156.

ISUANI, Ernesto, ROFMAN, Rafael y SAN MARTINO, Jorge (1996): Las jubilaciones del siglo XXI. ¿Podemos gastar a cuenta? En Boletín Informativo Techint, 286, pp.78-104.

JEFATURA DE GABINETE DE MINISTROS. PRESIDENCIA DE LA NACIÓN, 22 marzo 2017. Informe del Jefe de Gabinete de Ministros Lic. D. Marcos Peña a la Honorable Cámara de Diputados de la Nación. Informe Nº99.

MESSINA, Giuseppe (2014): Transformaciones y reformas en el sistema de pensiones argentino entre 2003 y 2013. Evaluando las politicas de inclusión más allá de lo contributivo. En Gestión y análisis de politicas públicas, (11), pp.1-14. Disponible en: https://revistasonline.inap.es/index.php/GAPP/article/view/10177 [fecha de consulta: 11 noviembre 2019].

PANIGO, Demian y MEDICI, Florencia (2013): El sistema de seguridad social en la Argentina. transformaciones recientes en perspectiva histórica. En Perspectiva sobre el Estado, las politicas públicas y la gestión, 1(1), pp.112-135. Disponible en: https://www.argentina.gob.ar/sites/default/files/inap_perspectivas_sobre_el_estado_las_politicas_publicas_y_la_gestion_vol_o1_nro_o1.pdf [fecha de consulta: 11 noviembre 2019]

PAYÁ, Fernando Horacio (h) y MARTÍN YÁÑEZ, María Teresa (2015): Régimen de Jubilaciones y Pensiones. Análisis crítico del Sistema Integrado Previsional Argentino (leyes 24.241 y 26.425) y regímenes especiales. $5^{\text {a }}$ Edición (Buenos Aires, Abeledo Perrot).

SALES, Antonio (2008): Reforma al Sistema Integrado de jubilaciones y Pensiones Argentino. En Revista Seguridad Social, 256, pp.71-92.

VACA EDGARDO Y VERITIER, María Gisela (2012): El fondo de garantía de sustentabilidad y la asignación universal por hijo. Oikonomos. En Revista científica de Ciencias Económicas, 1(2), 208-226.

\section{Jurisprudencia}

Chocobar, Sixto Celestino c/ Caja Nacional de Previsión para el Personal del Estado y Servicios Públicos s/ reajustes por movilidad (1996): Corte Suprema de la Nación Argentina XXVIII(C.278), 27 diciembre 1996. Disponible en: https://sjconsulta. csjn.gov.ar/sjconsulta/documentos/verUnicoDocumentoLink.html?idAnalisis=37 8ooo \& cache $=1576362680554$ [fecha de consulta: 14 diciembre 2019]. 
Sánchez, María del Carmen c. Administración Nacional de la Seguridad Social (2005): Corte Suprema de la Nación Argentina XXXVIII (S. 2758), 17 mayo 2005. Disponible en: https://sjconsulta.csjn.gov.ar/sjconsulta/documentos/verDocumentoSumario.html?idDocumentoSumario=11611. [fecha de consulta: 14 diciembre 2019].

Badaro, Adolfo Valentín c/ ANSeS s/ reajustes varios (2006): Corte Suprema de la Nación Argentina XLI (B.675), 8 de agosto de 2006. Disponible en: https://sjconsulta. csjn.gov.ar/sjconsulta/documentos/verDocumentoByIdLinksJSP.html?idDocume nto $=6066701 \&$ cache $=1576365548519$ [fecha de consulta: 14 diciembre 2019].

Badaro, Adolfo Valentín c/ ANSeS s/reajustes varios (2007): Corte Suprema de la Nación Argentina XLI (B.675), 26 noviembre 2007. Disponible en: https://sjconsulta. csjn.gov.ar/sjconsulta/documentos/verDocumentoByIdLinksJSP.html?idDocume nto $=6359051 \&$ cache $=1576365932854$ [fecha de consulta: 14 diciembre 2019] . 